\title{
Simulating Strategies for Improving Control of Hypertension Among Patients with Usual Source of Care in the United States: The Blood Pressure Control Model
}

\author{
Valy Fontil, $M D, M A S^{1,4}$, Kirsten Bibbins-Domingo, PhD, MD, MAS ${ }^{1,2,4}$, Dhruv S. Kazi, MD, MSc, MS ${ }^{2,5}$, \\ Stephen Sidney, MD, MPH', Pamela G. Coxson, $P h D^{1,4}$, Raman Khanna, MD, MAS ${ }^{6}$, \\ Ronald G. Victor, $\mathrm{MD}^{3}$, and Mark J. Pletcher, $M D, M P H^{2,1}$
}

'Division of General Internal Medicine, University of California San Francisco, San Francisco, CA, USA; ${ }^{2}$ Department of Epidemiology and
Biostatistics, University of California San Francisco, San Francisco, CA, USA; ${ }^{3}$ The Heart Institute/Hypertension Center, Cedars-Sinai Medical Center,
Los Angeles, CA, USA; ${ }^{4}$ UCSF Center for Vulnerable Populations at San Francisco General Hospital, San Francisco, CA, USA; ${ }^{5}$ Division of Cardiology,
San Francisco General Hospital, San Francisco, CA, USA; ${ }^{6}$ Division of Hospital Medicine, University of California San Francisco, San Francisco, CA, USA.

BACKGROUND: Only half of hypertensive adults achieve blood pressure (BP) control in the United States, and it is unclear how BP control rates may be improved most effectively and efficiently at the population level.

OBJECTIVE: We sought to compare the potential effects of system-wide isolated improvements in medication adherence, visit frequency, and higher physician prescription rate on achieving $\mathrm{BP}$ control at 52 weeks.

DESIGN: We developed a Markov microsimulation model of patient-level, physician-level, and system-level processes involved in controlling hypertension with medications. The model is informed by data from national surveys, cohort studies and trials, and was validated against two multicenter clinical trials (ALLHAT and VALUE).

SUBJECTS: We studied a simulated, nationally representative cohort of patients with diagnosed but uncontrolled hypertension with a usual source of care.

INTERVENTIONS: We simulated a base case and improvements of 10 and $50 \%$, and an ideal scenario for three modifiable parameters: visit frequency, treatment intensification, and medication adherence. Ideal scenarios were defined as $100 \%$ for treatment intensification and adherence, and return visits occurring within 4 weeks of an elevated office systolic BP.

MAIN OUTCOME: BP control at 52 weeks of follow-up was examined.

RESULTS: Among 25,000 hypothetical adult patients with uncontrolled hypertension (systolic BP $\geq 140$ mmHg), only $18 \%$ achieved BP control after 52 weeks using base-case assumptions. With 10/50 \%/idealized enhancements in each isolated parameter, enhanced treatment intensification achieved the greatest BP control (19/23/71 \%), compared with enhanced visit frequency (19/21/35 \%) and medication adherence (19/23/26\%). When all three processes were idealized, the model predicted a BP control rate of $95 \%$ at 52 weeks.

Electronic supplementary material The online version of this article (doi:10.1007/s11606-015-3231-8) contains supplementary material, which is available to authorized users.

Received August 22, 2014

Revised January 13, 2015

Accepted February 3, 2015

Published online March 7, 2015
CONCLUSION: Substantial improvements in BP control can only be achieved through major improvements in processes of care. Healthcare systems may achieve greater success by increasing the frequency of clinical encounters and improving physicians' prescribing behavior than by attempting to improve patient adherence to medications.

KEY WORDS: hypertension; blood pressure control; visit frequency; treatment intensification; medication adherence; microsimulation modeling.

J Gen Intern Med 30(8):1147-55

DOI: $10.1007 / \mathrm{s} 11606-015-3231-8$

(c) Society of General Internal Medicine 2015

\section{INTRODUCTION}

Hypertension is the most prevalent risk factor for cardiovascular disease nationally and internationally. ${ }^{1}$ Achieving blood pressure control (BP) in patients with hypertension reduces the risk of stroke and ischemic heart disease. ${ }^{2-4}$ Despite everincreasing public health resources dedicated to improving BP control, ${ }^{5-7}$ only half of the 78 million Americans with hypertension have their BP treated and controlled to the recommended value of below 140/90 mmHg. ${ }^{8}$ Under new BP targets recommended by the Panel Members Appointed to the Eighth Joint National Committee (JNC 8), ${ }^{9} \mathrm{BP}$ is controlled in less than $60 \%$ of hypertensive patients overall, and in less than $50 \%$ of patients younger than 60 years of age. ${ }^{10}$

Barriers to hypertension control occur at the levels of the patient, physician, and health system, and include insufficient access to high-quality care, physician and patient reluctance to intensify treatment for uncontrolled BP (i.e., inertia), and medication nonadherence. The relative importance of these different barriers is poorly understood, and is not systematically addressed by JNC 8. ${ }^{9}$ Although many different types of interventions have been shown to improve BP control in the population, ${ }^{11}$ there is little evidence on how population control rates can be improved most effectively and efficiently. Several clinical trials and integrated healthcare systems such as Kaiser 
Permanente and Veterans Health Administration have achieved high BP control rates (76-80 \%) by implementing multicomponent interventions, including improved access to quality care and medication-intensification protocols. ${ }^{12,13}$ However, quality improvement efforts in other clinical settings have failed to achieve similar success. ${ }^{5}$ A better understanding of the expected relative effectiveness of different types of interventions could inform public health efforts and facilitate a more cost-effective use of resources.

Microsimulation models can be used to project the potential population-level effects of healthcare interventions. ${ }^{14} \mathrm{We}$ developed the Blood Pressure (BP) Control Model as a tool to evaluate and compare the impact of patient-level, physicianlevel, and system-level interventions designed to improve management of hypertension across the U.S. adult population. The model integrates evidence from published observational and experimental studies with analyses of national survey data. We validated the model against two large hypertension control trials and then used the validated model to examine the effects of isolated improvements in three key parameters: frequency of clinic visits, the probability of treatment intensification at any visit where the recorded blood pressure is elevated, and medication adherence.

\section{METHODS}

\section{Conceptual Framework and Model Structure}

We developed the BP Control Model, an individual-level, state-transition Monte Carlo microsimulation model that simulates the basic sequence of healthcare processes involved in management of hypertension (Fig. 1). In each weekly cycle, individual patients may make a visit where blood pressure is measured (accounting for error/variability), the physician may prescribe a medication (with randomly varying systolic BPlowering effect), and the patient may become nonadherent to the last medication added. The base model uses the National Health and Nutrition Examination Survey (NHANES) study sample and incorporates NHANES sampling weights to simulate population of US adults with diagnosed but uncontrolled hypertension and a usual source of care. Key parameter inputs were derived from literature review and analyses of national survey data (Table 1). The model tracks systolic BP (SBP) over time, medication intensification steps, SBP-lowering effects from each intensification, and nonadherence events. The primary outcome for this analysis was BP control (percentage of patients with $\mathrm{SBP}<140 \mathrm{mmHg}$ ) at the end of 1 year.

\section{Definitions and Sources for Model Inputs}

We combined data from the Medical Expenditure Panel Survey (MEPS, 2003 to 2005), NHANES 1998 to 2008, and a previously reported multivariable visit interval analysis from an observational cohort study ${ }^{15}$ to estimate the frequency of clinic visits for persons with a usual source of care. Visit frequency varied by whether the patient had an elevated blood pressure, and whether a new medication had been prescribed at the most recent visit. Each visit represents an opportunity to measure blood pressure and intensify treatment. Physicians make management decisions based on BP measurements obtained during office visits. These measurements include true intra-individual BP variability over time, as well as random error in measurement. In order to simulate this complexity, we first modeled the "True SBP" using measurements from the NHANES data set. The "Office SBP" was then modeled by adding a random variability component to the True SBP derived from the distribution of visit-to-visit SBP variability observed in the European Lacidipine Study on Atherosclerosis. ${ }^{16}$

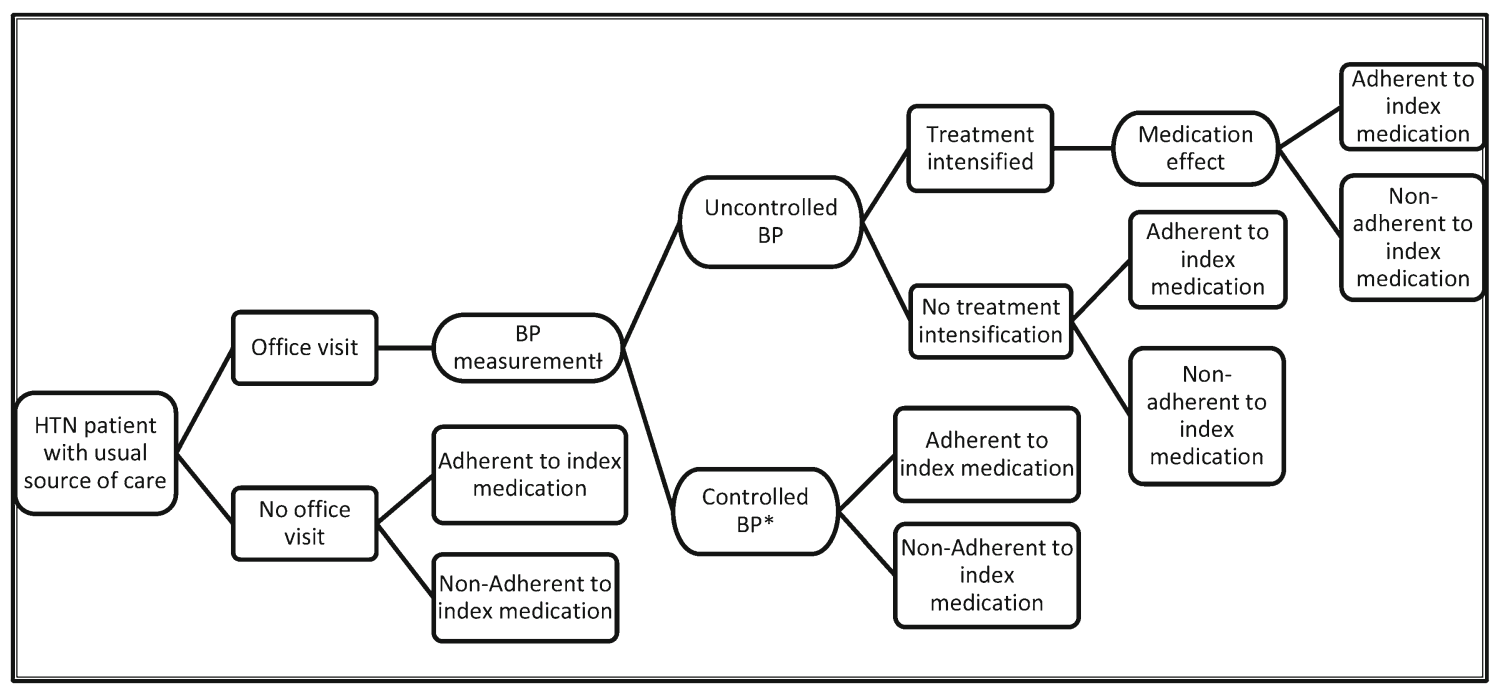

Figure 1. Model structure showing events that may occur in each weekly cycle. This figure represents the conceptual framework and model structure showing patient-level, physician-level, and system-level processes that are essential to controlling hypertension with medications. Abbreviations: HTN hypertension, BP blood pressure. * SBP $<140 \mathrm{mmHg}$. 
Table 1. Key Inputs, Parameters, and Sampling Distributions for Base-Case Model

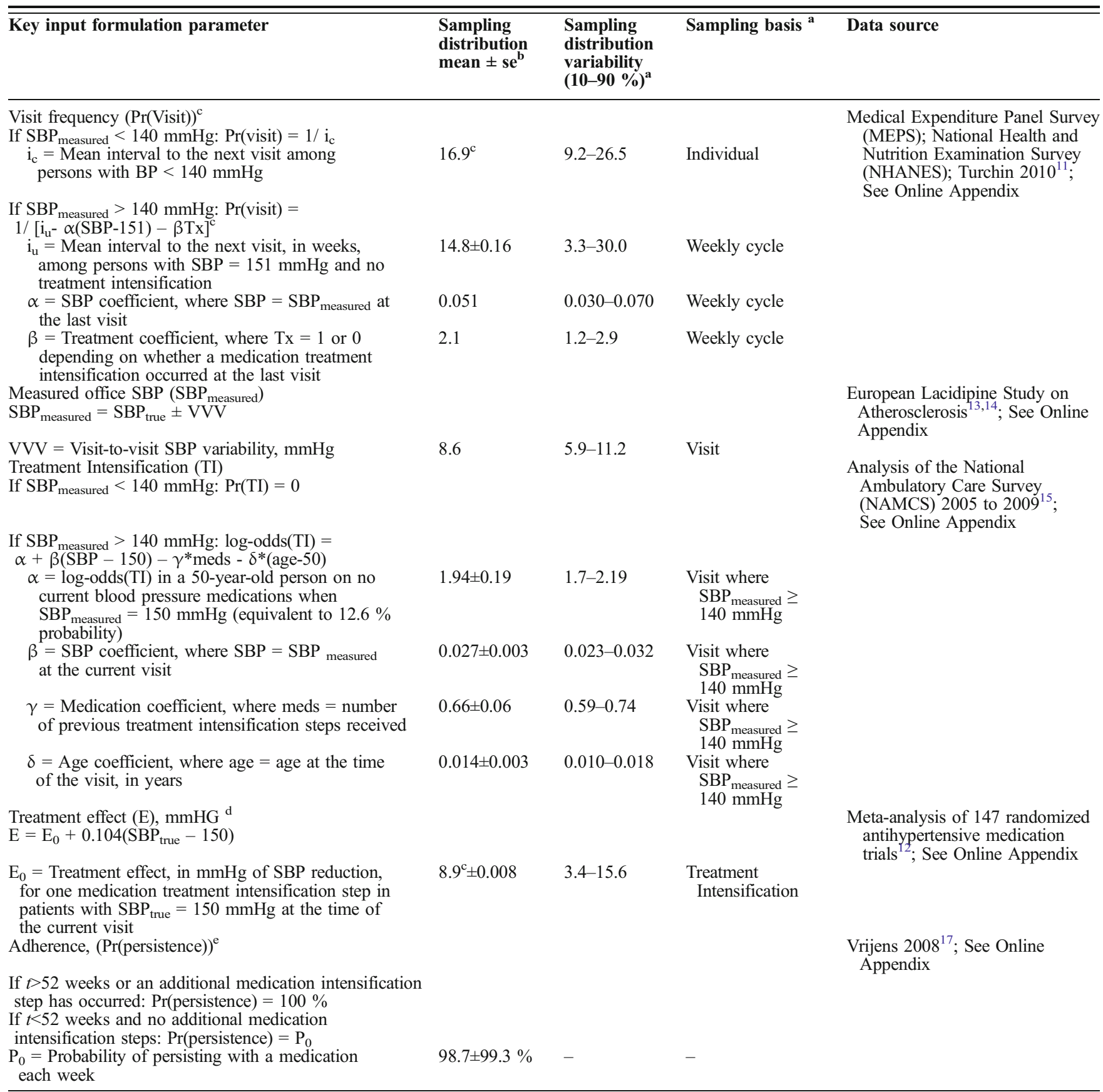

SBP Systolic blood pressure, SBP measured $O$ Office SBP measured at a visit and available for use in clinical decision-making, SBP true True SBP that is a cause of disease, modified by treatment, and measured with error at visits, Tx treatment intensification (yes=1, no=0), N/A not applicable, because the parameter value is constant and not sampled from a distribution

${ }^{a}$ In order to simulate between-person and within-person variability, most parameters are sampled from a distribution within each 25,000-person simulation. The Sampling Distribution Variability (10-90\%) describes the degree of variability, and Sampling Basis refers to the timing and frequency with which the sampling occurs during the simulation.

${ }^{b}$ For probabilistic sensitivity analyses, we added parameter uncertainty (presented here as the standard error of the mean for that parameter) in 500 "outer loop" iterations (each with 25,000 hypothetical patients) for several key parameters; see Methods for details

${ }^{c}$ Visit Frequency is defined as the probability of making an office visit in any given week, or Pr(visit)

${ }^{d}$ Reduction in $S B P_{\text {true }}$ from one treatment intensification step, which is assumed to be equivalent to adding one drug at half the standard dose ${ }^{12}$

eAdherence is assumed to refer to persistence in the use of a medication (rather than "execution", which is the \% of pills taken during use), and is modeled on a weekly basis such that persistence at 1 year $=50 \%{ }^{17}$ Online Appendix

When the physician observes an elevated Office SBP (defined in the base case as SBP $\geq 140 \mathrm{mmHg}$ ), she may increase the dose of the existing medication or add a new medication ("treatment intensification"). The probability of treatment intensification was estimated from a published analysis of visitbased physician prescribing using the National Ambulatory 
Medical Care Survey ${ }^{17}$ (see Appendix) that accounts for the higher likelihood of a prescription with fewer current BP medications, higher measured SBP, and younger age (Table 1).

True SBP is then reduced when a treatment intensification event occurs. The degree of SBP reduction for any given treatment intensification event is simulated by sampling from a distribution of treatment effects for adding one medication at half-standard dose derived from a meta-analysis of 147 randomized antihypertensive drug trials. ${ }^{18}$

In the interval between visits, a patient may discontinue the BP medication, with the resultant reversal of any gains achieved from the last treatment intensification. Medication adherence is comprised of "execution"19 (taking $100 \%$ of medication doses as prescribed) and "persistence" (ongoing use of the medication). ${ }^{19}$ Based on an analysis of 21 phase IV clinical trials of antihypertensive medications ${ }^{20}$ we assumed a $50 \%$ persistence at 1 year in the base case. We assumed that imperfect execution (i.e., missed doses) is captured in the average treatment intensification effect and did not separately model this parameter.

The Online Appendix provides additional detail on the methods, parameters, and derivations thereof.

\section{Model Validation}

We validated the model against the intervention arms of two large, multicenter clinical trials: the Antihypertensive and Lipid-Lowering Treatment to Prevent Heart Attack Trial $\left(\right.$ ALLHAT) ${ }^{21}$ and the Valsartan Antihypertensive Long-term Use Evaluation (VALUE). ${ }^{22}$ For the purpose of the validation, we generated hypothetical ALLHAT and VALUE study populations by sampling from NHANES participants with hypertension in survey years corresponding to the trial's study period, applying study inclusion and exclusion criteria, and then adjusting NHANES survey weights in order to approximate the mean and standard deviation of the baseline SBP distribution reported in each of the two clinical trials. In the first cycle of the simulation, we discontinued all prerandomization BP medications and used the equation for medication effect described above to estimate the SBP prior to starting any study medication. We then modeled the trial interventions by modifying our base case assumptions for visit frequency and treatment intensification to match the protocol and reported visit frequency and treatment intensification for each trial (Table 2; see Online Appendix for details). We then compared the simulated results to the $\mathrm{BP}$ control rates observed in the trials. Base case assumptions for all other parameters, including medication adherence, medication dose effect size, and SBP visit-to-visit variability, were not modified for the purpose of the validation.

\section{Model Application: 1-Year Simulation of Interventions to Control BP}

We simulated three interventions to improve BP control in the base-case population: visit frequency, treatment intensification, and medication adherence. After estimating BP control with base-case values, we estimated the impact of $10 \%$ improvement, $50 \%$ improvement, and an "idealized" scenario for each parameter, defined as: A return visit occurring within 4 weeks of an elevated office SBP; $100 \%$ treatment intensification at every visit with an elevated Office SBP; and $100 \%$ adherence. We chose 10 and $50 \%$ scenarios to represent a marginal and significant improvement, respectively. We then simulated three combination interventions in which we combined 1) $10 \%$ improvements, 2) $50 \%$ improvements, and 3) "idealized values" for all three parameters.

We incorporated the variability in most parameters into each simulation iteration - in the "inner loop"-at the level of the individual patient, weekly cycle, or visit, to capture the many stochastic and random processes that occur during the process of BP control (Table 1). This inter-individual variation is counterbalanced, to some extent, by the large hypothetical sample size we chose $(25,000)$, which stabilizes populationlevel results, such as the BP control rate. To estimate imprecision in our results from parameter uncertainty, we performed a probabilistic sensitivity analysis incorporating variation in several key parameters in 500 "outer loop" iterations (each with 25,000 hypothetical patients), and present the $95 \%$ confidence interval of the BP Control difference from this sensitivity analysis in our results. Parameter uncertainty incorporated into the probabilistic sensitivity analysis is presented in Table 1 as the standard error of the sampling distribution mean.

We used TreeAge software (TreeAge Software, Inc.) for all simulations.

\section{RESULTS}

The NHANES-derived hypothetical ALLHAT and VALUE study populations closely matched the actual trial study populations in terms of age and blood pressure distribution. Model-predicted BP control rates were similar to observed control rates in the intervention arms of the respective studies at 1 year $(58.7 \%$ simulated vs. $55.1 \%$ observed in ALLHAT and $56.2 \%$ simulated vs. $52.2 \%$ observed in VALUE; Table 2).

Using base case assumptions and the NHANES-derived sample of persons with uncontrolled hypertension in the US, the model predicted that $18 \%$ of uncontrolled hypertensive patients with a usual source of care would achieve BP control at 52 weeks. Control rates were higher among men, young adults, those not on BP medications at baseline, and patients with lower baseline SBP (Table 3).

Simulated interventions leading to improvements in visit frequency, treatment intensification and adherence led to higher control rates (Table 4). An isolated $10 \%$ improvement in each of these parameters produced small improvements in BP control. Fifty percent improvements in each of the parameters achieved higher rates of BP control $(21,23$, and $23 \%$ for 
Table 2. Model Validation Comparing Simulated and Observed BP Control Rates for ALLHAT and VALUE Clinical Trials

\begin{tabular}{|c|c|c|c|c|c|c|}
\hline & \multicolumn{2}{|c|}{ ALLHAT } & \multicolumn{4}{|c|}{ VALUE } \\
\hline $\begin{array}{l}\text { Inclusion criteria } \\
\text { Duration of follow-up }\end{array}$ & \multicolumn{2}{|c|}{$\begin{array}{l}\text { A randomized, double-blind, active-controlled clinical } \\
\text { trial of } 33,357 \text { high-risk adult patients with } \\
\text { hypertension in } 623 \text { North American centers } \\
\text { comparing the effects of Chlorthalidone, Amlodipine, } \\
\text { and Lisinopril on reducing cardiovascular outcomes } \\
\text { Men and women age } \geq 55 \text { years who had stage I or } \\
\text { stage II HTN with } \geq 1 \text { additional CVD risk factor* } \\
5 \text { years }\end{array}$} & \multicolumn{4}{|c|}{ 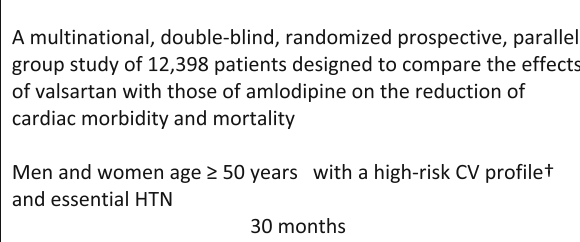 } \\
\hline Assumptions/model adjustments & Observed & Simulated & \multicolumn{3}{|c|}{ Observed } & Simulated \\
\hline Visit frequency & $\begin{array}{l}\text { Scheduled follow-up at } 1,3, \\
6,9, \text { and } 12 \text { months with } \\
91.7 \% \text { visit adherence at } 1 \\
\text { year }\end{array}$ & $\begin{array}{l}91.7 \% \text { probability of } \\
\text { attendance for each } \\
\text { scheduled visit at } 1,3 \text {, } \\
6,9 \text {, and } 12 \text { months }\end{array}$ & \multicolumn{3}{|c|}{$\begin{array}{l}\text { Monthly for the first } 5 \text { visits, followed } \\
\text { by semi-annual visits }\end{array}$} & \multirow{7}{*}{$\begin{array}{l}\text { Monthly for the first } \\
\text { visits, followed by } \\
\text { visits at weeks } 26 \text { and } \\
52 \\
\text { Probability of Tlई: } \\
\text { Step 1: } 100 \% \\
\text { Step 2:: } 98.8 \% \% \\
\text { Step 3: } 98.1 \% \\
\text { Step 4: } 97.1 \% \\
\text { step 5: } 94.8 \% \\
\text { other: default model } \\
\text { assumption }\end{array}$} \\
\hline \multirow{7}{*}{ Treatment intensification $(T I)^{\ddagger}$} & \multirow{7}{*}{\multicolumn{2}{|c|}{$\begin{array}{l}\text { Not reported } \\
\text { Protocol: Step 1: titrate } \\
\text { assigned study drug } \\
\text { Step 2: add open-label } \\
\text { agents "when necessary" to } \\
\text { goal BP <140/90 } \mathrm{mmHg} \text {. }\end{array}$}} & \begin{tabular}{|l|}
$\begin{array}{l}\text { Treatment } \\
\text { steps } \\
\text { completed }\end{array}$ \\
\end{tabular} & $\begin{array}{l}\% \text { study } \\
\text { population }\end{array}$ & \begin{tabular}{|c|}
$\%$ \\
uncontro \\
lled BP \\
\end{tabular} & \\
\hline & & & 1 & 23.9 & 6.0 & \\
\hline & & & 2 & 39.7 & 5.9 & \\
\hline & & & $3 \& 4$ & 26.6 & 11.7 & \\
\hline & & & 5 & 15.1 & Not used & \\
\hline & & & Other & 18.6 & Not used & \\
\hline & & & $\begin{array}{l}\text { Step 1 }=\text { initia } \\
\text { Step 2 = up-ti } \\
\text { Step 3 = addit } \\
\text { Step 4 = up-ti } \\
\text { Step 5 = free } \\
\text { Other }=\text { more }\end{array}$ & $\begin{array}{l}\text { tion of monot } \\
\text { tration of mor } \\
\text { tion of thiazide } \\
\text { tration of thia } \\
\text { add-on } \\
\text { TI or non-pro }\end{array}$ & $\begin{array}{l}\text { lerapy } \\
\text { otherapy } \\
\text { ide } \\
\text { ocol }\end{array}$ & \\
\hline Medication adherence & $\begin{array}{l}\text { Mean adherence not } \\
\text { reported }\end{array}$ & $\begin{array}{l}\text { Default model } \\
\text { assumption }\end{array}$ & Mean adhere & nce not repor & & $\begin{array}{l}\text { Default model } \\
\text { assumption }\end{array}$ \\
\hline \multirow{2}{*}{ Baseline characteristics ${ }^{\#}$} & \multicolumn{2}{|c|}{ ALLHAT } & \multicolumn{4}{|c|}{ VALUE } \\
\hline & Observed & Simulated & & Observed & & Simulated \\
\hline Age & $66.9(7.7)$ & $66.2(10.2)$ & & $67.2(8.1)$ & & $68.7(10.9)$ \\
\hline SBP & $146.3(15.7)$ & $146.8(15.6)$ & & $154.7(19.0)$ & & $154.8(19.5)$ \\
\hline BP Control & $27.4 \%$ & $28.8 \%$ & & $21.9 \%$ & & $21.1 \%$ \\
\hline \multicolumn{7}{|l|}{ Outcomes } \\
\hline Time point & 1 year & 52 weeks & & 1 year & & 52 weeks \\
\hline Final SBP & $138.2(16.3)$ & $135.7(11.7)$ & & $141(16.0)$ & & $138.3(13.4)$ \\
\hline Change in SBP & Not reported & $7.5(10.4)$ & & ot reported & & $11.8(14.2)$ \\
\hline \multicolumn{7}{|l|}{ BP Control } \\
\hline Definition & $\mathrm{BP}<140 / 90$ & $\mathrm{SBP}<140$ & & $\mathrm{SBP}<140$ & & $\mathrm{SBP}<140$ \\
\hline Rate & $55.1 \%$ & $58.7 \%$ & & $52.2 \%$ & & $55.8 \%$ \\
\hline
\end{tabular}

Abbreviations: $B P=$ blood pressure, $S B P=$ systolic blood pressure, $f / u=$ follow up

Observed and simulated outcomes are aggregated for the entire cohorts in the ALLHAT and VALUE trials

*Cardiovascular risk factors: diabetes, cigarette smoking, hypercholesterolemia, left ventricular hypertrophy, chronic kidney disease, and stroke.

tBased on an algorithm of cardiovascular risk factors.

I Probability of dose increase or addition of new medication in visits where the BP is above target. We used the model's default assumption in protocol steps with open-label add-on and inadequate data for estimation of TI. We estimated the incidence of treatment intensification (TI) in each step by algebraic calculations using the trial's reported drug utilization data summarized in the table. ALHAT was designed to compare first-line antihypertensive therapies and left choice and titration of additional medications in large part to physicians' discretion.

${ }^{s}$ Probability of $T I=\left(\frac{100-\text { sum of } \% \text { study population at previous steps }}{100-\text { sum of } \% \text { study population at previous steps }+\% \text { uncontrolled BP }}\right)^{\frac{1}{\text { remaining } \# \text { of visits }}}$

${ }^{\#}$ Simulations were done using NHANES-derived cohorts calibrated to approximate the baseline SBP distribution for each study trial; see Methods.

visit frequency, treatment intensification and adherence), and substantially larger and more variable effects when these processes were enhanced to ideal levels. Control was achieved in $71 \%$ of patients with ideal treatment intensification, representing an increase of $53 \%$ (95\% Confidence Interval (CI): 49-56) from the base-case scenario; $35 \%$ with ideal visit frequency (17\% increase, $95 \%$ CI: $15-18)$ and $26 \%$ with ideal adherence ( $8 \%$ increase, $95 \% \mathrm{CI}$ : $7-10)$. In a supplemental simulation, modeling a $50 \%$ probability of intensifying treatment when the Office SBP is elevated, with base case assumptions for the other two parameters, led to $43 \% \mathrm{BP}$ control. With combination interventions, 10 and $50 \%$ improvements in all three parameters led to 21 and $32 \%$ achieving BP control at 1 year; idealization of all three processes resulted in $95 \%$ control.

\section{DISCUSSION}

Our study suggests that substantial improvements in BP control will require major improvements in processes of care. Near-universal control of BP is attainable within a year when all processes of care are set at optimal levels. Incremental improvements in the different parameters that determine BP control, either in isolation or combination, led to variable incremental improvements in BP control. Healthcare systems 
Table 3. Simulated Process and Outcome Measures for Base-Case Population by Patient Characteristics

\begin{tabular}{|c|c|c|c|c|c|c|c|c|c|}
\hline \multicolumn{3}{|c|}{ Baseline characteristics } & \multicolumn{5}{|c|}{ Process measures over 52 weeks of simulated follow-up } & \multicolumn{2}{|c|}{ Outcomes at 52 weeks } \\
\hline & \multirow[t]{2}{*}{$N(\%)$} & \multirow{2}{*}{$\begin{array}{l}\text { Mean } \\
\text { Systolic } \\
\text { blood } \\
\text { pressure, } \\
\text { mmHG } \\
\text { (SD) }\end{array}$} & \multirow{2}{*}{$\begin{array}{l}\text { Mean } \\
\text { number } \\
\text { of office } \\
\text { visits } \\
\text { (SD) }\end{array}$} & \multicolumn{3}{|c|}{ Treatment intensification (TI) } & \multirow{2}{*}{$\begin{array}{l}\begin{array}{l}\text { Medication } \\
\text { non-adherence }\end{array} \\
\begin{array}{l}\text { Incident } \\
\text { discontinuation } \\
\text { of medication }\end{array}\end{array}$} & \multirow[b]{2}{*}{$\begin{array}{l}\text { Mean SBP } \\
\text { reduction, } \\
\text { mmHg } \\
\text { (SD) }\end{array}$} & \multirow[b]{2}{*}{$\begin{array}{l}\text { BP } \\
\text { Control } \\
\text { at } 52 \\
\text { weeks }\end{array}$} \\
\hline & & & & $\begin{array}{l}N(\%) \\
\text { receiving at } \\
\text { least one TI } \\
\text { for elevated } \\
\text { office } \mathrm{SBP} \\
(\geq 140 \mathrm{mmHg}) \\
\text { over } 52 \text { weeks }\end{array}$ & $\begin{array}{l}\text { Mean total } \\
\text { occurrence } \\
\text { of treatment } \\
\text { intensification } \\
\text { (SD) }\end{array}$ & $\begin{array}{l}\text { Incident } \\
\text { TI in } \\
\text { visits with } \\
\text { elevated } \\
\text { office SBP }\end{array}$ & & & \\
\hline Overall & 25,000 & $\begin{array}{c}153.2 \\
(14.4)\end{array}$ & $3.9(2.4)$ & $\begin{array}{l}16,512 \\
(18.3 \%)\end{array}$ & $0.7(0.8)$ & 0.22 & 0.31 & $6.1(10.0)$ & 0.18 \\
\hline \multicolumn{10}{|l|}{ Gender } \\
\hline Male & $\begin{array}{r}12,746 \\
(51.0)\end{array}$ & $\begin{array}{c}151.7 \\
(13.4)\end{array}$ & $3.9(2.3)$ & $\begin{array}{l}8,609 \\
(19.7 \%)\end{array}$ & $0.7(0.8)$ & 0.24 & 0.31 & $6.4(10.3)$ & 0.22 \\
\hline Female & $\begin{array}{r}12,254 \\
(49.0)\end{array}$ & $\begin{array}{c}154.9 \\
(15.6)\end{array}$ & $4.0(2.4)$ & $\begin{array}{l}7,903 \\
(17.0 \%)\end{array}$ & $0.6(0.8)$ & 0.20 & 0.31 & $5.7(9.6)$ & 0.15 \\
\hline \multicolumn{10}{|l|}{ Age (years) } \\
\hline $18-39$ & $\begin{array}{r}2,147 \\
(8.6)\end{array}$ & $\begin{array}{c}149.7 \\
(10.3)\end{array}$ & $3.8(2.2)$ & $\begin{array}{l}1,862 \\
(27.7 \%)\end{array}$ & $0.9(0.8)$ & 0.35 & 0.29 & $8.3(11.1)$ & 0.32 \\
\hline $41-59$ & $\begin{array}{c}8,387 \\
(33.5)\end{array}$ & $\begin{array}{c}151.6 \\
(14.5)\end{array}$ & $3.9(2.3)$ & $\begin{array}{l}5,981 \\
(21.3 \%)\end{array}$ & $0.7(0.8)$ & 0.26 & 0.30 & $6.8(10.4)$ & 0.23 \\
\hline $60-80$ & $\begin{array}{r}14,466 \\
(57.9)\end{array}$ & $\begin{array}{c}154.8 \\
(15.0)\end{array}$ & $4.0(2.4)$ & $\begin{array}{l}8,669 \\
(15.6 \%)\end{array}$ & $0.6(0.8)$ & 0.19 & 0.32 & $5.3(9.4)$ & 0.19 \\
\hline \multicolumn{10}{|c|}{ True SBP (mmHg) at baseline } \\
\hline $140-159$ & $\begin{array}{c}19,482 \\
(77.9)\end{array}$ & $\begin{array}{r}146.8 \\
(5.4)\end{array}$ & $3.8(2.2)$ & $\begin{array}{l}10,376 \\
(17.1 \%)\end{array}$ & $0.5(0.7)$ & 0.22 & 0.32 & $5.2(9.4)$ & 0.23 \\
\hline $160-179$ & $\begin{array}{l}3,839 \\
(15.4)\end{array}$ & $\begin{array}{c}168.3 \\
(5.8)\end{array}$ & $4.4(2.6)$ & $\begin{array}{l}3,709 \\
\quad(18.7 \%)\end{array}$ & $1.0(0.9)$ & 0.20 & 0.31 & $7.2(9.5)$ & 0.03 \\
\hline$\geq 180$ & $1,679(6.7)$ & $\begin{array}{c}193.6 \\
(11.4)\end{array}$ & $4.8(2.7)$ & $\begin{array}{l}2,427 \\
(25.2 \%)\end{array}$ & $1.4(1.0)$ & 0.27 & 0.27 & $13.4(13.0)$ & 0.006 \\
\hline \multicolumn{10}{|c|}{ \# of BP meds at baseline } \\
\hline 0 & $\begin{array}{c}8,205 \\
(32.8)\end{array}$ & $\begin{array}{c}150.8 \\
(12.8)\end{array}$ & $3.9(2.3)$ & $\begin{array}{l}5,972 \\
(22.1 \%)\end{array}$ & $0.7(0.8)$ & 0.24 & 0.27 & $7.2(10.6)$ & 0.25 \\
\hline 1 & $\begin{array}{c}7,590 \\
(30.4)\end{array}$ & $\begin{array}{c}154.9 \\
(15.8)\end{array}$ & $4.0(2.4)$ & $\begin{array}{l}6,720 \\
(24.6 \%)\end{array}$ & $0.9(0.8)$ & 0.32 & 0.32 & $8.0(11.0)$ & 0.23 \\
\hline 2 & $\begin{array}{c}5,116 \\
(20.5)\end{array}$ & $\begin{array}{c}154.3 \\
(16.3)\end{array}$ & $4.0(2.4)$ & 2,618 (13.6\%) & $0.5(0.7)$ & 0.17 & 0.33 & $4.5(8.7)$ & 0.12 \\
\hline$\geq 3$ & $\begin{array}{c}4,089 \\
(16.4)\end{array}$ & $\begin{array}{c}153.9 \\
(12.6)\end{array}$ & $4.0(2.4)$ & $\begin{array}{l}1,202 \\
(7.2 \%)\end{array}$ & $0.3(0.5)$ & 0.10 & 0.39 & $2.2(5.9)$ & 0.06 \\
\hline
\end{tabular}

BP blood pressure

$B P$ control $=$ proportion of patients with systolic $B P<140 \mathrm{mmHg}$

may achieve greater success by focusing resources on increasing the frequency of clinical encounters (e.g., visits) and improving physicians' prescribing behavior than by attempting to improve patient adherence.

Computer simulation modeling has been instrumental in developing clinical guidelines and policy in general, ${ }^{14,23-26}$ and for hypertension in particular. ${ }^{27}$ Farley and colleagues ${ }^{27}$ used mathematical modeling to project the potential mortality benefits of increasing hypertension control, while Turner and Schalkwyk ${ }^{28}$ used computer simulation of BP variability to examine spurious identification of hypertension in clinical studies. Our model represents a considerable advance over previous models, because it simultaneously models multiple processes involved in BP management, thus permitting the comparison of different types of multifaceted interventions for management of hypertension. The model is informed by data from national surveys, cohort studies and trials, and predicted BP control rates similar to those achieved in the intervention arms of two multicenter clinical trials (ALLHAT and VALUE).
Our finding that treatment intensification rates strongly impact BP control is consistent with previous reports. ${ }^{11,29,30}$ Several systematic reviews have shown that protocols that require high visit frequency with vigorous treatment intensification have the greatest impact on BP control. ${ }^{11,31,32}$ National initiatives in the U.S aim to disseminate hypertension management protocols that primarily focus on enhancing treatment intensification in patients with $\mathrm{BP}$ above targets. ${ }^{33}$ Since there are legitimate reasons why a clinician may choose not to intensify treatment for an elevated SBP measured in the office, the optimal/attainable rate of treatment intensification is unclear. However, real-world evaluation of practice patterns suggests frequent missed opportunities for appropriate treatment intensification in management of hypertension. ${ }^{17,34-37}$ The large impact of treatment intensification on population BP control seen in our model argues for large-scale adoption of interventions that have been shown to increase treatment intensification rates. ${ }^{36,38-41}$

Our results suggest that visit frequency is an important parameter in the management of hypertension. Although 
Table 4. Simulated Blood Pressure Control at 52 Weeks Under Different Intervention Scenarios

\begin{tabular}{|c|c|c|c|c|}
\hline \multirow[t]{2}{*}{ Scenarios } & \multirow{2}{*}{$\begin{array}{l}\text { Parameter } \\
\text { values }\end{array}$} & \multicolumn{3}{|l|}{ Outcomes } \\
\hline & & $\begin{array}{l}\text { \% SBP control } \\
(\mathrm{SBP}<140 \mathrm{mmHg})\end{array}$ & $\begin{array}{l}\text { Change in } \% \mathrm{SBP} \\
\text { control compared to } \\
\text { base case }\left(95 \% \mathrm{CI}^{* *}\right)\end{array}$ & $\begin{array}{l}\text { SBP reduction, } \\
\text { mmHg (SD) }\end{array}$ \\
\hline Base case $^{\mathrm{a}}$ & & $18.3 \%$ & & $6.1(10.0)$ \\
\hline \multicolumn{5}{|l|}{ Visit frequency ${ }^{b}$} \\
\hline Base case average visit rate (visits/week) & 0.068 & $18.3 \%$ & - & - \\
\hline $10 \%$ increase $^{\mathrm{c}}$ & 0.074 & $19.4 \%$ & $+1.1 \%(0.9-1.3 \%)$ & $6.3(10.2)$ \\
\hline $50 \%$ increase $^{c}$ & 0.101 & $21.1 \%$ & $+2.8 \%(2.3-3.3 \%)$ & $7.3(10.8)$ \\
\hline $\begin{array}{l}\text { Ideal (probability of visit within } 4 \text { weeks after an } \\
\text { elevated office sbp) }\end{array}$ & 0.707 & $34.8 \%$ & $+16.5 \%(15.2-17.7 \%)$ & $12.8(13.9)$ \\
\hline \multicolumn{5}{|l|}{ Treatment intensification $(\mathrm{TI})^{\mathrm{d}}$, per visit with elevated BP } \\
\hline Base case average probability of treatment intensification & 0.18 & $18.3 \%$ & - & - \\
\hline $10 \%$ increase $^{\mathrm{c}}$ & 0.19 & $19.4 \%$ & $+1.1 \%(0.9-1.3 \%)$ & $6.4(10.1)$ \\
\hline $50 \%$ increase $^{\mathrm{c}}$ & 0.23 & $23.1 \%$ & $+4.8 \%(4.4-5.3 \%)$ & $7.6(11.0)$ \\
\hline Ideal (probability of TI) & 1 & $71.0 \%$ & $+52.7 \%(49.0-56.2 \%)$ & $23.4(17.6)$ \\
\hline \multicolumn{5}{|l|}{ Medication adherence, } \\
\hline Base case adherence rate ${ }^{\mathrm{f}}$, per year( 52 weeks $)$ & 0.50 & $18.3 \%$ & - & - \\
\hline $10 \%$ increase $^{\mathrm{c}}$ & 0.55 & $19.1 \%$ & $+0.9 \%(0.7-1.1 \%)$ & $6.4(10.1)$ \\
\hline $50 \%$ increase $^{c}$ & 0.75 & $22.5 \%$ & $+4.2 \%(3.5-5.0 \%)$ & $7.1(10.4)$ \\
\hline Ideal & 1 & $26.3 \%$ & $+8.1 \%(6.7-9.5 \%)$ & $8.5(11.2)$ \\
\hline \multicolumn{5}{|l|}{ Combination interventions } \\
\hline Base case & & $18.3 \%$ & - & - \\
\hline $10 \%$ increase in all parameters ${ }^{\mathrm{g}}$ & - & $21.0 \%$ & $+2.7 \%(2.3-3.2 \%)$ & $7.0(10.7)$ \\
\hline $50 \%$ increase in all parameters ${ }^{\mathrm{h}}$ & - & $31.9 \%$ & $+13.6 \%(12.1-15.1 \%)$ & $10.4(12.3)$ \\
\hline Ideal values in all parameters ${ }^{\mathrm{i}}$ & - & $95.1 \%$ & $+76.8 \%(73.1-80.4 \%)$ & $31.9(18.5)$ \\
\hline
\end{tabular}

SBP systolic blood pressure

${ }^{a}$ Base case: all three parameters at base-case (current practice) values (Table 1)

${ }^{b}$ Probability of a visit in any given week. We only increased this probability in the weeks following an elevated sbp $(\geq 140 \mathrm{mmHg})$

${ }^{c} 10$ and $50 \%$ improvements modeled by multiplying the visit rate, odds of treatment intensification, and cumulative adherence proportion by a factor of

1.1 and 1.5. Rates and odds are then converted to the probabilities that are used as model inputs for each parameter

${ }^{d}$ Probability of treatment intensification at a visit where BP is elevated

${ }^{e}$ Probability of ongoing use of the last prescribed medication at 52 weeks

${ }^{f}$ Medication adherence is the cumulative proportion of patients continuing to take their last prescribed medication 52 weeks after it was prescribed. We convert this rate to a weekly probability of ongoing medication use, assuming a constant rate of medication discontinuation over 52 weeks, for use in the model

${ }^{g} 10 \%$ improvements in all three parameters

${ }^{h} 50 \%$ improvements in all three parameters

${ }^{i}$ All three parameters at the Ideal value, defined as: a return occurring visit within 4 weeks of an elevated office SBP; $100 \%$ treatment intensification at every visit with an elevated Office SBP; and $100 \%$ adherence

** -95\% confidence interval, derived from probabilistic sensitivity analyses varying key parameters

JNC $8^{9}$ did not address visit frequency, our simulation of ideal visit frequency is based on the JNC 7 guideline that patients are followed up within a month when an elevated BP is noted. ${ }^{42}$ This frequency of follow-up is seldom achieved in current practice, ${ }^{15,43}$ but its implementation in our model nearly doubled BP control in the population (from 18 to $35 \%$ ) at 1 year. Previous studies suggest that "effective" visit frequency may be improved with the help of allied health professionals, telephone BP monitoring, and ambulatory BP monitoring. ${ }^{11,44-46}$

Our findings are consistent with previous publications demonstrating a relatively weak and inconsistent relationship between medication adherence and BP control. ${ }^{7,30,41,47}$ Vigen and colleagues ${ }^{47}$ compared clinic-level medication adherence and treatment intensification as potential clinical performance measures using the Cardiovascular Research Network Hypertension Registry including 162,879 patients in 89 clinics. Treatment intensification was associated with BP control at 12 months, but there was no significant association between medication adherence and BP control at the clinic level. In a secondary analysis of 819 patients from seven clinics affiliated with a safety net hospital, Rose and colleagues ${ }^{30}$ found that treatment intensification was associated with similar BP improvement regardless of a patient's level of adherence. In our simulation, idealized visit frequency and treatment intensification led to greater improvements in BP control rate than improvements attained by optimizing adherence. At the individual level, clinicians' knowledge or suspicion of patient medication nonadherence has been cited as a reason for not intensifying treatment. ${ }^{30,48}$ However, our simulation is consistent with previous evidence that treatment intensification improves BP control even among patients with sub-optimal medication adherence. ${ }^{30,41}$ The effect of adherence interventions to improve population BP control may be limited, because most established patients with hypertension are at least partially adherent to their antihypertensive regimen. ${ }^{6,30}$

Our finding of near-universal control of hypertension (95\%) within a year by optimizing visit frequency, treatment intensification, and medication adherence would be difficult to achieve in the "real world," largely because optimizing all three factors would itself be extremely difficult. Nevertheless, the Veterans Health Affairs had achieved an impressive improvement in BP control (from 46 to $76 \%$ ), while Kaiser 
Permanente has seen an increase from $44 \%$ to greater than $80 \%$ as a result of interventions that primarily worked through these modifiable factors. ${ }^{12,13}$

As with all models, the BP Control Model is a simplification of reality, and several specific limitations are worth noting. The model simulates the systolic and not the diastolic BP, even though both values may affect treatment decisions in practice. However, systolic BP is an equal or stronger predictor of cardiovascular risk in adults older than 49 years, and is more difficult to control than diastolic BP. ${ }^{22,42,49}$ The model does not account for differences in SBP lowering effect, adherence, or side effects specific to different drug classes. Because increasing encounter frequency may produce not only more opportunities for treatment intensification, but also improved treatment adherence, ${ }^{50}$ our model likely underestimated the impact of frequent visits on BP control. Recent guidelines vary in their recommendations for the appropriate BP target for some elderly patients; ${ }^{9,51}$ our model does not attempt to modify BP targets for different individuals (SBP target is $<140 \mathrm{mmHg}$ for all individuals). Other individual-level characteristics that might modify processes of care, such as race/ethnicity and access to care, have not been incorporated into this version of the model. Despite these limitations, the BP Control model represents a careful synthesis of evidence and national data yielding results that appear to have good external and face validity.

\section{CONCLUSION}

The US Department of Health and Human Services Million Hearts initiative aims to prevent 1 million myocardial infarctions and strokes through nationwide adoption of evidencebased hypertension treatment protocols to help achieve a control rate of $65 \%$ by $2017 .{ }^{6,33}$ However, BP control in the U.S is improving by only $1 \%$ per year. ${ }^{8,33}$ Our study suggests that substantial improvements in BP control can only be achieved through major improvements in processes of care; increasing the frequency of clinical encounters and improving physicians' prescribing behavior (in isolation or combination) would have a greater impact on BP control than attempts to boost patients' medication adherence. The BP Control Model can help researchers and healthcare leaders, prioritize interventional approaches, tailor management protocols in particular populations, and help us find ways to meet our public health objectives for management of hypertension in the United States.

Acknowledgements: Funding: This work was supported and funded by the UCSF Center for Vulnerable Populations at San Francisco General Hospital (NIMHD grant 1P60MD006902, NINDS grant U54NS081760, and supplement to U54NS081760), UCSF Primary Care Research Fellowship (NRSA grant T3HP19025), R01HL117983 (RGV), and the Lincy Foundation.

Conflicts of Interest: Dr. Ronald Victor is an advisor on the Scientific Advisory Board of Northwind Medical, Inc. All other authors report no conflicts of interest.
Corresponding Author: Valy Fontil, MD, MAS; UCSF Center for Vulnerable Populations at San Francisco General Hospital, San Francisco, CA, USA (e-mail: valy.fontil@ucsf.edu).

\section{REFERENCES}

1. Yang 9, Cogswell ME, Flanders WD, et al. Trends in cardiovascular health metrics and associations with all-cause and CVD mortality among US adults. JAMA. 2012;307(12):1273-83.

2. Staessen JA, Fagard $\mathbf{R}$, Thijs $\mathbf{L}$, et al. Randomised double-blind comparison of placebo and active treatment for older patients with isolated systolic hypertension. The Systolic Hypertension in Europe (Syst-Eur) Trial Investigators. Lancet. 1997;350(9080):757-64.

3. Beckett NS, Peters R, Fletcher AE, et al. Treatment of hypertension in patients 80 years of age or older. N Engl J Med. 2008;358(18): 1887-98.

4. Prevention of stroke by antihypertensive drug treatment in older persons with isolated systolic hypertension. Final results of the Systolic Hypertension in the Elderly Program (SHEP). SHEP Cooperative Research Group. JAMA. 1991;265(24):3255-3264.

5. Landon BE, Hicks LS, O'Malley AJ, et al. Improving the management of chronic disease at community health centers. N Engl J Med. 2007;356(9):921-34

6. Fung V, Huang J, Brand R, Newhouse JP, Hsu J. Hypertension treatment in a medicare population: adherence and systolic blood pressure control. Clin Ther. 2007;29(5):972-84.

7. LaFleur J, Nelson RE, Sauer BC, Nebeker JR. Overestimation of the effects of adherence on outcomes: a case study in healthy user bias and hypertension. Heart. 2011;97(22):1862-9.

8. Egan BM, Zhao Y, Axon RN. US trends in prevalence, awareness, treatment, and control of hypertension, 1988-2008. JAMA. 2010;303(20):2043-50.

9. James PA, Oparil S, Carter BL, et al. 2014 evidence-based guideline for the management of high blood pressure in adults: report from the panel members appointed to the Eighth Joint National Committee (JNC 8). JAMA. 2014;311(5):507-20.

10. Navar-Boggan AM, Pencina MJ, Williams K, Sniderman AD, Peterson ED. Proportion of US adults potentially affected by the 2014 hypertension guideline. JAMA. 2014;311(14): 1424-9.

11. Glynn LG, Murphy AW, Smith SM, Schroeder K, Fahey T. Interventions used to improve control of blood pressure in patients with hypertension. Cochrane Database Syst Rev. 2010;3:CD005182.

12. Jaffe MG, Lee GA, Young JD, Sidney S, Go AS. Improved blood pressure control associated with a large-scale hypertension program. JAMA. 2013;310(7):699-705.

13. Fletcher RD, Amdur RL, Kolodner R, et al. Blood pressure control among US veterans: a large multiyear analysis of blood pressure data from the Veterans Administration health data repository. Circulation. 2012;125(20):2462-8.

14. Abraham JM. Using microsimulation models to inform U.S. health policy making. Health Serv Res. 2013;48(2 Pt 2):686-95.

15. Turchin A, Goldberg SI, Shubina M, Einbinder JS, Conlin PR. Encounter frequency and blood pressure in hypertensive patients with diabetes mellitus. Hypertension. 2010;56(1):68-74.

16. Mancia G, Facchetti R, Parati G, Zanchetti A. Visit-to-visit blood pressure variability, carotid atherosclerosis, and cardiovascular events in the European Lacidipine Study on Atherosclerosis. Circulation. 2012;126(5):569-78.

17. Khanna RR, Victor RG, Bibbins-Domingo K, Shapiro MF, Pletcher MJ. Missed opportunities for treatment of uncontrolled hypertension at physician office visits in the United States, 2005 through 2009. Arch Intern Med. 2012;172(17):1344-5.

18. Law MR, Morris JK, Wald NJ. Use of blood pressure lowering drugs in the prevention of cardiovascular disease: meta-analysis of 147 randomised trials in the context of expectations from prospective epidemiological studies. BMJ. 2009;338:b1665.

19. Hill MN, Miller NH, DeGeest S. American Society of Hypertension Writing G. ASH position paper: Adherence and persistence with taking medication to control high blood pressure. J Clin Hypertens (Greenwich). 2010;12(10):757-64.

20. Vrijens B, Vincze G, Kristanto P, Urquhart J, Burnier M. Adherence to prescribed antihypertensive drug treatments: longitudinal study of electronically compiled dosing histories. BMJ. 2008;336(7653):1114-7. 
21. Officers A, Coordinators for the ACRGTA, Lipid-Lowering Treatment to Prevent Heart Attack T. Major outcomes in high-risk hypertensive patients randomized to angiotensin-converting enzyme inhibitor or calcium channel blocker vs. diuretic: The Antihypertensive and Lipid-Lowering Treatment to Prevent Heart Attack Trial (ALLHAT). JAMA. 2002;288(23):2981-2997.

22. Julius S, Kjeldsen SE, Brunner H, et al. VALUE trial: Long-term blood pressure trends in 13,449 patients with hypertension and high cardiovascular risk. Am J Hypertens. 2003; 16(7):544-8.

23. Glied S, Tilipman N. Simulation modeling of health care policy. Annu Rev Public Health. 2010;31:439-55.

24. Weinstein MC, Toy EL, Sandberg EA, et al. Modeling for health care and other policy decisions: uses, roles, and validity. Value Health. 2001;4(5):348-61.

25. Mandelblatt JS, Cronin KA, Bailey S, et al. Effects of mammography screening under different screening schedules: model estimates of potential benefits and harms. Ann Intern Med. 2009;151(10):738-47.

26. Kristensen AH, Flottemesch TJ, Maciosek MV, et al. Reducing Childhood Obesity through U.S. Federal Policy: A Microsimulation Analysis. Am J Prev Med. 2014.

27. Farley TA, Dalal MA, Mostashari F, Frieden TR. Deaths preventable in the U.S. by improvements in use of clinical preventive services. Am J Prev Med. 2010;38(6):600-9.

28. Turner MJ, van Schalkwyk JM. Blood pressure variability causes spurious identification of hypertension in clinical studies: a computer simulation study. Am J Hypertens. 2008;21(1):85-91.

29. Daugherty SL, Powers JD, Magid DJ, et al. The association between medication adherence and treatment intensification with blood pressure control in resistant hypertension. Hypertension. 2012;60(2):303-9.

30. Rose AJ, Berlowitz DR, Manze M, Orner MB, Kressin NR. Intensifying therapy for hypertension despite suboptimal adherence. Hypertension. 2009;54(3):524-9.

31. Holder KK. Interventions to improve blood pressure control in patients with hypertension. Am Fam Physician. 2007;76(3):373-4.

32. Fahey T, Schroeder K, Ebrahim S. Interventions used to improve control of blood pressure in patients with hypertension. Cochrane Database Syst Rev. 2006;2:CD005182.

33. Frieden TR, King SM, Wright JS. Protocol-based treatment of hypertension: a critical step on the pathway to progress. JAMA. 2014;311(1):21-2.

34. Okonofua EC, Simpson KN, Jesri A, Rehman SU, Durkalski VL, Egan BM. Therapeutic inertia is an impediment to achieving the Healthy People 2010 blood pressure control goals. Hypertension. 2006;47(3):345-51.

35. Berlowitz DR, Ash AS, Hickey EC, et al. Inadequate management of blood pressure in a hypertensive population. N Engl J Med. 1998;339(27): 1957-63.

36. Phillips LS, Branch WT, Cook CB, et al. Clinical inertia. Ann Intern Med. 2001;135(9):825-34.

37. Fontil V, Pletcher M, Khanna R, Guzman D, Victor R, Bibbins-Domingo K Physician Underutilization of Effective Medications for Resistant
Hypertension at Office Visits in the United States: NAMCS 2006-2010. Journal of General Internal Medicine. 2013:1-9.

38. Davis BR, Ford CE, Remington RD, Stamler R, Hawkins CM. The Hypertension Detection and Follow-up Program design, methods, and baseline characteristics and blood pressure response of the study population. Prog Cardiovasc Dis. 1986;29(3 Suppl 1):11-28.

39. Selby JV, Uratsu CS, Fireman B, et al. Treatment intensification and risk factor control: toward more clinically relevant quality measures. Med Care. 2009;47(4):395-402.

40. Stewart S, Carrington MJ, Swemmer $\mathbf{C H}$, et al. Effect of intensive structured care on individual blood pressure targets in primary care: multicentre randomised controlled trial. BMJ. 2012;345:e7156.

41. Magid DJ, Ho PM, Olson KL, et al. A multimodal blood pressure control intervention in 3 healthcare systems. Am J Manag Care. 2011;17(4):e96103.

42. Chobanian AV, Bakris GL, Black HR, et al. The Seventh Report of the Joint National Committee on Prevention, Detection, Evaluation, and Treatment of High Blood Pressure: the JNC 7 report. JAMA. 2003;289(19):2560-72.

43. Guthmann R, Davis N, Brown M, Elizondo J. Visit frequency and hypertension. J Clin Hypertens (Greenwich). 2005;7(6):327-32.

44. Clark CE, Smith LF, Taylor RS, Campbell JL. Nurse led interventions to improve control of blood pressure in people with hypertension: systematic review and meta-analysis. BMJ. 2010;341:c3995.

45. Morgado MP, Morgado SR, Mendes LC, Pereira LJ, Castelo-Branco M. Pharmacist interventions to enhance blood pressure control and adherence to antihypertensive therapy: Review and meta-analysis. Am J Health Syst Pharm. 2011;68(3):241-53.

46. Rader F, Elashoff RM, Niknezhad S, Victor RG. Differential treatment of hypertension by primary care providers and hypertension specialists in a barber-based intervention trial to control hypertension in Black men. Am J Cardiol. 2013;112(9):1421-6.

47. Vigen R, Shetterly S, Magid DJ, et al. A comparison between antihypertensive medication adherence and treatment intensification as potential clinical performance measures. Circ Cardiovasc Qual Outcomes. 2012;5(3):276-82

48. Manze M, Rose AJ, Orner MB, Berlowitz DR, Kressin NR. Understanding racial disparities in treatment intensification for hypertension management. J Gen Intern Med. 2010;25(8):819-25.

49. Franklin SS, Larson MG, Khan SA, et al. Does the relation of blood pressure to coronary heart disease risk change with aging? The Framingham Heart Study. Circulation. 2001;103(9):1245-9.

50. Patel NC, Crismon ML, Miller AL, Johnsrud MT. Drug adherence: effects of decreased visit frequency on adherence to clozapine therapy. Pharmacotherapy. 2005;25(9):1242-7.

51. Hypertension EETFftMoA. 2013 Practice guidelines for the management of arterial hypertension of the European Society of Hypertension (ESH) and the European Society of Cardiology (ESC): ESH/ESC Task Force for the Management of Arterial Hypertension. J Hypertens. 2013;31(10):1925-38. 